\title{
UNCERTAINTIES IN ATOMIC DATA AND THEIR PROPAGATION THROUGH SPECTRAL MODELS. I.
}

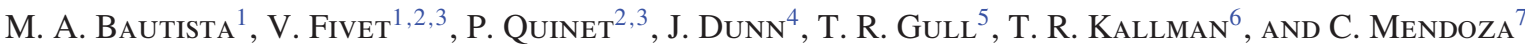 \\ ${ }^{1}$ Department of Physics, Western Michigan University, Kalamazoo, MI 49008, USA; manuel.bautista@wmich.edu \\ ${ }^{2}$ Astrophysique et Spectroscopie, Université de Mons-UMONS, B-7000 Mons, Belgium \\ ${ }^{3}$ IPNAS, Université de Liège, Sart Tilman, B-4000 Liège, Belgium \\ ${ }^{4}$ Physical Science Department, Georgia Perimeter College, Dunwoody, GA 30338, USA \\ ${ }^{5}$ Code 667, NASA Goddard Space Flight Center, Greenbelt, MD 20771, USA \\ ${ }^{6}$ Code 662, NASA Goddard Space Flight Center, Greenbelt, MD 20771, USA \\ ${ }^{7}$ Centro de Física, Instituto Venezolano de Investigaciones Científicas (IVIC), P.O. Box 20632, Caracas 1020A, Venezuela \\ Received 2013 January 4; accepted 2013 April 22; published 2013 May 20
}

\begin{abstract}
We present a method for computing uncertainties in spectral models, i.e., level populations, line emissivities, and emission line ratios, based upon the propagation of uncertainties originating from atomic data. We provide analytic expressions, in the form of linear sets of algebraic equations, for the coupled uncertainties among all levels. These equations can be solved efficiently for any set of physical conditions and uncertainties in the atomic data. We illustrate our method applied to spectral models of O III and Fe II and discuss the impact of the uncertainties on atomic systems under different physical conditions. As to intrinsic uncertainties in theoretical atomic data, we propose that these uncertainties can be estimated from the dispersion in the results from various independent calculations. This technique provides excellent results for the uncertainties in $A$-values of forbidden transitions in $[\mathrm{Fe} \mathrm{II}]$.
\end{abstract}

Key words: atomic data - atomic processes - line: formation - methods: data analysis - molecular data molecular processes - techniques: spectroscopic

Online-only material: color figures

\section{INTRODUCTION}

Much can be learned about the behavior and evolution of an astronomical source through analysis of properly calibrated spectra. Interpretation can lead to estimates of density and temperature conditions, the chemical composition, the dynamics, and the sources of energy that power the emitting object. Such an interpretation requires modeling, with sufficiently high accuracy, the excitation and ionization balance of plasmas out of local thermodynamic equilibrium (LTE). But ultimately, the accuracy of the models depends on the quality of the atomic/molecular data employed.

At present, atomic data exist for most spectral lines observed from the infrared to the X-rays. These data account for most processes leading to tens of thousands of transitions from all ionic stages of nearly all elements of the first five rows of the periodic table. However, this huge amount of data has been obtained primarily through theoretical calculations with only sparse checks with experimental measurements.

Despite many advances in spectral modeling, mostly in terms of increased completeness and improved quality of atomic/ molecular parameters, there is yet no general method to quantify the uncertainties in atomic data and how these propagate through spectral models. Astronomers seek to understand such uncertainties and how they affect their analysis of observations. Thus, international conferences have been organized in recent years to discuss these issues, see Luridiana et al. (2011) and Luridiana \& García-Rojas (2012). In recent years, a few authors have presented methods based on the Monte Carlo numerical technique for propagating uncertainties through spectral models, e.g., Wesson et al. (2012) and Ballance et al. (2013). These techniques are powerful, in the sense that they allow one to explore various different scenarios, such as correlated uncertainties in atomic data and asymmetric uncertainty distributions. However, Monte Carlo techniques are numerically complex and inefficient. In the context of spectral models, the Monte Carlo method consists in solving the population balance equation for stochastic variations of each of the atomic parameters in the model. In a 5-level spectral model of O III, there are 19 nonvanishing atomic parameters among $A$-values and excitation rate coefficients for a fixed temperature. In a 52-level model of Fe II, like the one adopted here, there are over 2031 non-vanishing atomic rates to consider for every temperature. A typical Fe II model that accounts for Ly $\alpha$ involves $\sim 800$ levels and over 60,000 atomic parameters. Statistically meaningful results from stochastic techniques would require sampling $\sim 100$ variations of each atomic parameter. Thus, Monte Carlo studies need to solve the population balance equations between a few thousand times and several hundreds of thousands of times depending on the size of the atomic model. It is impractical to perform such a task on a regular basis for every ion and spectrum that is analyzed.

Finding a general and efficient method for estimating uncertainties in spectral models would be useful for two reasons. First, the accuracy of atomic/molecular data must be known before reliable conclusions can be put forward on physically realistic comparisons between theoretical and observed spectra. At present researchers can only provide best fits to observed spectra without much understanding of the uncertainties impacting the results. Just to cite one example, in the study of broad absorption line quasars (e.g., Dunn et al. 2010) researchers seek to fit observed spectra with results of photoionization modeling codes to deduce the hydrogen column density and ionization parameter of the absorbing cloud. In addition, absorption column densities of metastable levels of a few ions are used as density diagnostics. From these parameters the distances of the absorbing clouds 

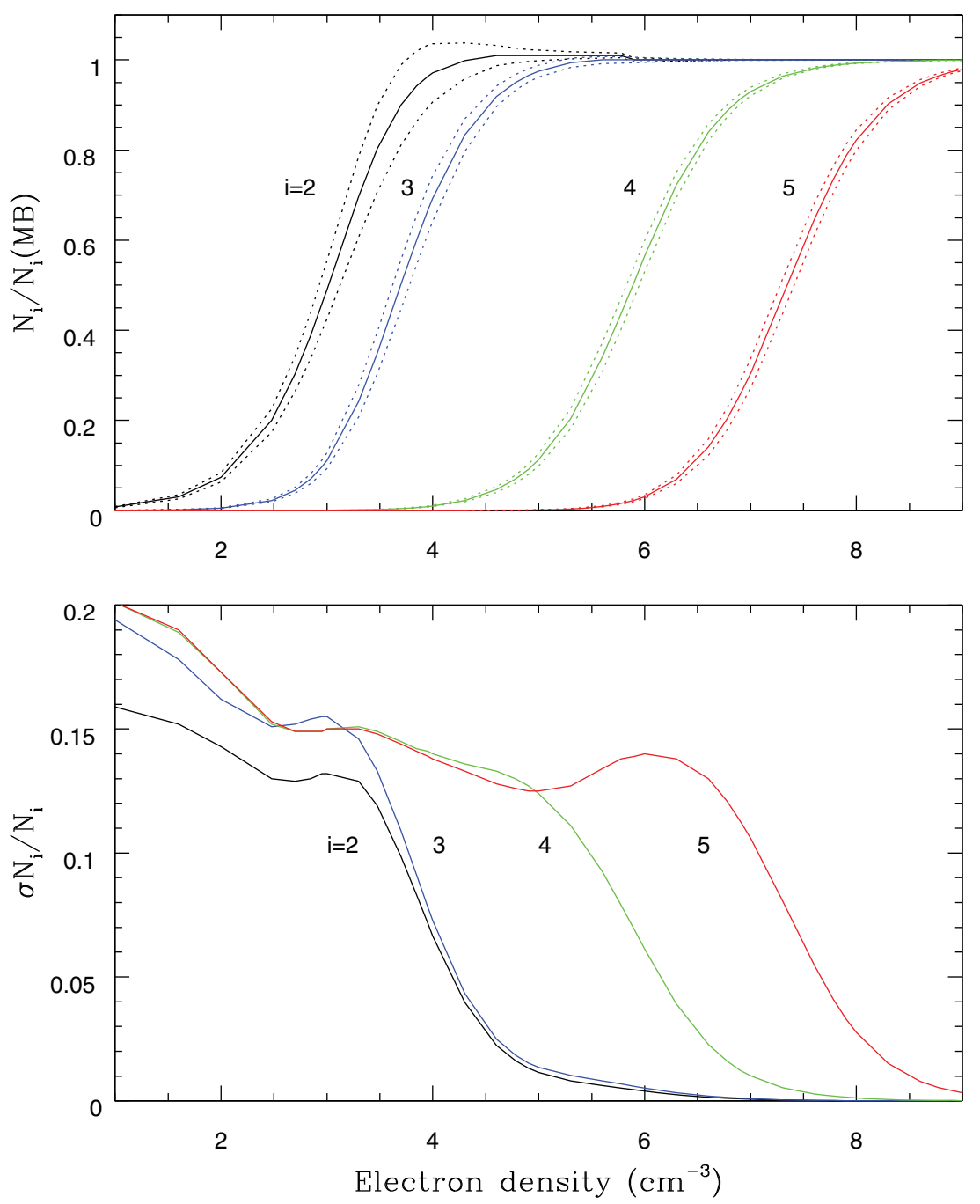

Figure 1. Level populations relative to their Boltzmann limits (upper panel) and relative level population uncertainties (lower panel) for the $2 p^{2}{ }^{3} P_{1}(i=2),{ }^{3} P_{2}$ $(i=3),{ }^{1} D_{2}(i=4)$, and ${ }^{1} S_{0}(i=5)$ excited levels of O III.

(A color version of this figure is available in the online journal.)

from the central engine are estimated, which in turn allows one to compute kinetic luminosities, mass accretion rates, and more. But, ionization parameters and density diagnostics can be very uncertain. Many studies have to rely on a selected group of lines in fitting the spectrum, and density diagnostics are based on either a single line ratio or, even worse, on two or three diagnostics that seem to disagree with each other. The second motivation for studying error propagation through spectral models is that homogeneously accurate atomic data cannot be obtained for all transitions of a complex and/or very large atomic system; for instance systems with multiple metastable levels (e.g., Fe II) or models with hundreds of energy levels such as those needed in UV and X-ray spectroscopy. In such models, error propagation analysis of the spectrum could discriminate between a few critically important atomic transitions and the very large numbers of less consequential transitions. Conversely, detailed error analysis could direct further theoretical and/or experimental efforts to selectively obtain specific atomic measures that would significantly improve spectral models, instead of trying to determine all possible rates at once.

This paper is organized as follows. Section 2 presents the analytical solution to the uncertainties in level populations of a non-LTE spectral model for assumed uncertainties in atomic parameters. In Section 3 we propose a mechanism to estimate the uncertainties in atomic/molecular data and we test in the case of Fe II through extensive comparisons with observed spectra. In Section 4 we discuss the uncertainties in line emissivities and emission line ratio diagnostics. Section 5 presents our conclusions. For the sake of clarity, the rest of the paper deals explicitly with the case of population balance by electron impact excitation followed by spontaneous radiative decay. It is also assumed that the plasma is optically thin. However, we note that our method can easily be extended to ionization balance computations, to additional excitation mechanisms such as continuum and Bowen fluorescence, and to optically thick transitions.

\section{UNCERTAINTIES IN LEVEL POPULATIONS AND COLUMN DENSITIES}

Under steady-state balance the population, $N_{i}$, of a level $i$ is given by

$$
N_{i}=\frac{\sum_{k \neq i} N_{k}\left(n_{e} q_{k, i}+A_{k, i}\right)}{n_{e} \sum_{j \neq i} q_{i, j}+\sum_{j<i} A_{i, j}},
$$



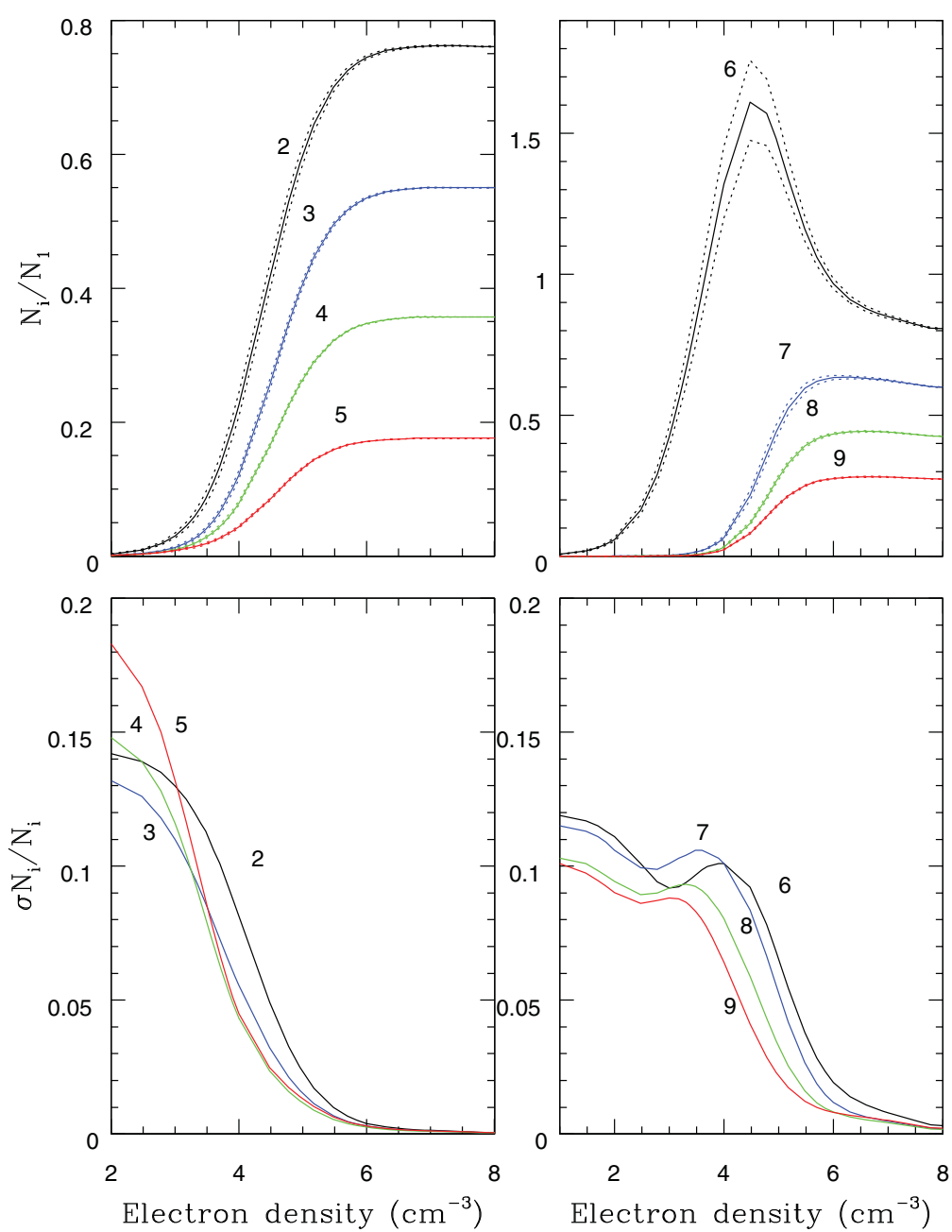

Figure 2. Level populations relative to the ground level (upper panels) and relative level population uncertainties (lower panels) for the $3 d^{6} 4 s^{6} D_{7 / 2}(i=2)$, ${ }^{6} D_{5 / 2}$ $(i=3),{ }^{6} D_{3 / 2}(i=4),{ }^{6} D_{1 / 2}(i=5), 3 d^{7}{ }^{4} F_{9 / 2}(i=6), 3 d^{7}{ }^{4} F_{7 / 2}(i=7), 3 d^{7}{ }^{4} F_{5 / 2}(i=8)$, and $3 d^{7}{ }^{4} F_{3 / 2}(i=9)$ of Fe II.

(A color version of this figure is available in the online journal.)

where $n_{e}$ is the electron density, $A_{k, i}$ is the Einstein spontaneous radiative rate from level $k$ to level $i$, and $q_{k, i}$ is the electron impact transition rate coefficient for transitions from level $k$ to level $i$. Here, we assume that the electron velocity distribution follows the Maxwell-Boltzmann function, thus $q_{k, i}$ and $q_{i, k}$ are both proportional to a symmetrical effective collision strength, $\Upsilon_{k, i}=\Upsilon_{i, k}$, which is the source of uncertainty in the collisional transition rates. Assuming that the spectral model is arranged in increasing level energy order, $A_{k, i}=0$ whenever $k<i$. We note that the second term in the denominator of the above equation is the inverse of the lifetime of level $i$, i.e., $\tau_{i}=\left(\sum_{j<i} A_{i, j}\right)^{-1}$. It may be pointed out that lifetimes are generally dominated by a few strong transitions, which are much more accurately determined than for the weak transitions. Thus, $\tau_{i}$ carries smaller uncertainties than individual $A$-values. Then, Equation (1) can be written as

$$
N_{i}=\frac{\sum_{k \neq i} N_{k}\left(n_{e} q_{k, i}+A_{k, i}\right)}{n_{e} \tau_{i} \sum_{j \neq i} q_{i, j}+1} \tau_{i}=\frac{\sum_{k \neq i} N_{k}\left(n_{e} q_{k, i}+A_{k, i}\right)}{n_{e} / n_{i}^{c}+1} \tau_{i},
$$

where $n_{i}^{c}$ is the so-called critical density of level $i$ and is defined as $n_{i}^{c}=\left(\tau_{i} \sum_{j \neq i} q_{i, j}\right)^{-1}$.

Since the population balance equations are linear, the propagation of uncertainties through level populations is also linear. Thus, without loss of accuracy, the uncertainty in the population of level $i, \delta N_{i}$, can be computed as

$$
\begin{aligned}
\left(\delta N_{i}\right)^{2}= & \sum_{k \neq i}\left[\left(\frac{\partial N_{i}}{\partial \Upsilon_{k, i}}\right)^{2}\left(\delta \Upsilon_{k, i}\right)^{2}+\left(\frac{\partial N_{i}}{\partial A_{k, i}}\right)^{2}\left(\delta A_{k, i}\right)^{2}\right] \\
& +\sum_{j \neq i}\left(\frac{\partial N_{i}}{\partial A_{i, j}}\right)^{2}\left(\delta A_{i, j}\right)^{2}+\sum_{k \neq i}\left(\frac{\partial N_{i}}{\partial N_{k}}\right)^{2}\left(\delta N_{k}\right)^{2} .
\end{aligned}
$$

The first three terms on the right-hand side of this equation represent the direct propagation of uncertainties from atomic rates to or from level $i$. The last term in the equation correlates the uncertainty in level $i$ with the uncertainties in the level populations of all other levels that contribute to it. Then,

$$
\begin{aligned}
& \left(\frac{\delta N_{i}}{N_{i}}\right)^{2}-\sum_{k \neq i} N_{k}^{2} \frac{\left(n_{e} q_{k, i}+A_{k, i}\right)^{2}}{\kappa^{2}}\left(\frac{\delta N_{k}}{N_{k}}\right)^{2} \\
& =\frac{1}{\kappa^{2}}\left[n_{e}^{2} \sum_{k \neq i}\left(N_{k} q_{k, i}-N_{i} q_{i, k}\right)^{2}\left(\frac{\delta \Upsilon_{k, i}}{\Upsilon_{k, i}}\right)^{2}\right. \\
& \left.+\sum_{k>i}\left(N_{k} A_{k, i}\right)^{2}\left(\frac{\delta A_{k, i}}{A_{k, i}}\right)^{2}+\left(\frac{N_{i}^{2}}{\tau^{2}}\right)^{2}\left(\frac{\delta \tau_{i}}{\tau_{i}}\right)^{2}\right]
\end{aligned}
$$

where $\kappa_{i}=\sum_{k \neq i} N_{k}\left(n_{e} q_{k, i}+A_{k, i}\right)$. 
This linear set of equations yields the uncertainties in the populations of all levels. Before proceeding to solve these equations it is worth pointing out some properties: (1) Uncertainties are obtained relative to the computed level populations regardless of the normalization adopted for these. This is important because while some spectral models compute populations relative to the ground level other models solve for normalized populations such that $\sum N_{k}$ is either 1 or the total ionic abundance. However, the equation above is generally applicable regardless of the normalization adopted. (2) In the high density limit, $n_{e} \rightarrow \infty$, the right-hand side of the equation goes to zero, thus the population uncertainties naturally go to zero as the populations approach the Maxwell-Boltzmann values (LTE conditions). (3) By having an analytical expression for the propagation of uncertainties one can do a detailed analysis of the spectral model to identify the key atomic data that determine the quality of the model for any plasma conditions. (4) The set of linear equations for the uncertainties needs to be solved only once for any set of conditions and the system is of the same size as that for the level populations. This is unlike Monte Carlo approaches that require solving population balance equations hundreds of times, which makes real-time computation of uncertainties impractical.

The set of equations above can be readily solved by writing them as

$$
B \bar{x}=\bar{b},
$$

where $x_{i}=\left(\delta N_{i} / N_{i}\right)^{2}$, and the matrix and vector elements of $B$ and $\bar{b}$ are given by Equation (2).

Figure 1 shows the populations and population uncertainties for the first four excited levels of $\mathrm{O}$ III as a function of the electron density at a temperature of $10^{4} \mathrm{~K}$. For this computation we have assumed uncertainties of $5 \%$ in the lifetimes, $10 \%$ in individual $A$-values, and $20 \%$ in the effective collision strengths. The levels considered here are $2 p^{2}{ }^{3} P_{0,1,2},{ }^{1} D_{2}$, and ${ }^{1} S_{0}$. It is seen that levels 2 through 5 have maximum uncertainties, $\sim 20 \%$, in the low-density limit where the populations are determined by collisional excitations from the ground level. As the electron density increases, thermalization of levels with similar energies and radiative cascades start becoming more important, which diminishes the contribution of uncertainties in collision strengths and enhances the importance of uncertainties in $A$-values. For high densities all population uncertainties naturally go to zero as the populations approach the Boltzmann limit. Furthermore, the population uncertainties exhibit multiple contributions and peaks as the metastable levels ${ }^{3} P_{1}$ and ${ }^{3} P_{2}$ become populated and their uncertainties propagate through higher levels.

Figure 2 shows the populations, relative to the ground level, and population uncertainties for the first eight excited levels of Fe II as a function of the electron density at a temperature of $10^{4} \mathrm{~K}$. For these calculations we use atomic data from Bautista \& Pradhan (1998) and assume uncertainties of 5\% in the lifetimes, $10 \%$ in individual $A$-values, and $20 \%$ in the effective collision strengths. The levels considered here are $3 d^{6} 4 s^{5} D_{9 / 2,7 / 2,5 / 2,3 / 2}$ and $3 d^{7}{ }^{4} F_{9 / 2,7 / 2,5 / 2,3 / 2}$. An interesting characteristic of the Fe II system is that the $3 d^{7}{ }^{4} F_{9 / 2}$ excited level is more populated, at least according to the atomic data adopted here, than the ground level at densities around $10^{4} \mathrm{~cm}^{-3}$ typical of $\mathrm{H}$ II regions. Moreover, under these conditions only $\sim 20 \%$ of the total Fe II abundance is in the ground level. This implies that, unlike lighter species, where excitation is dominated by the ground level or the ground multiplet, in Fe II all metastable levels are

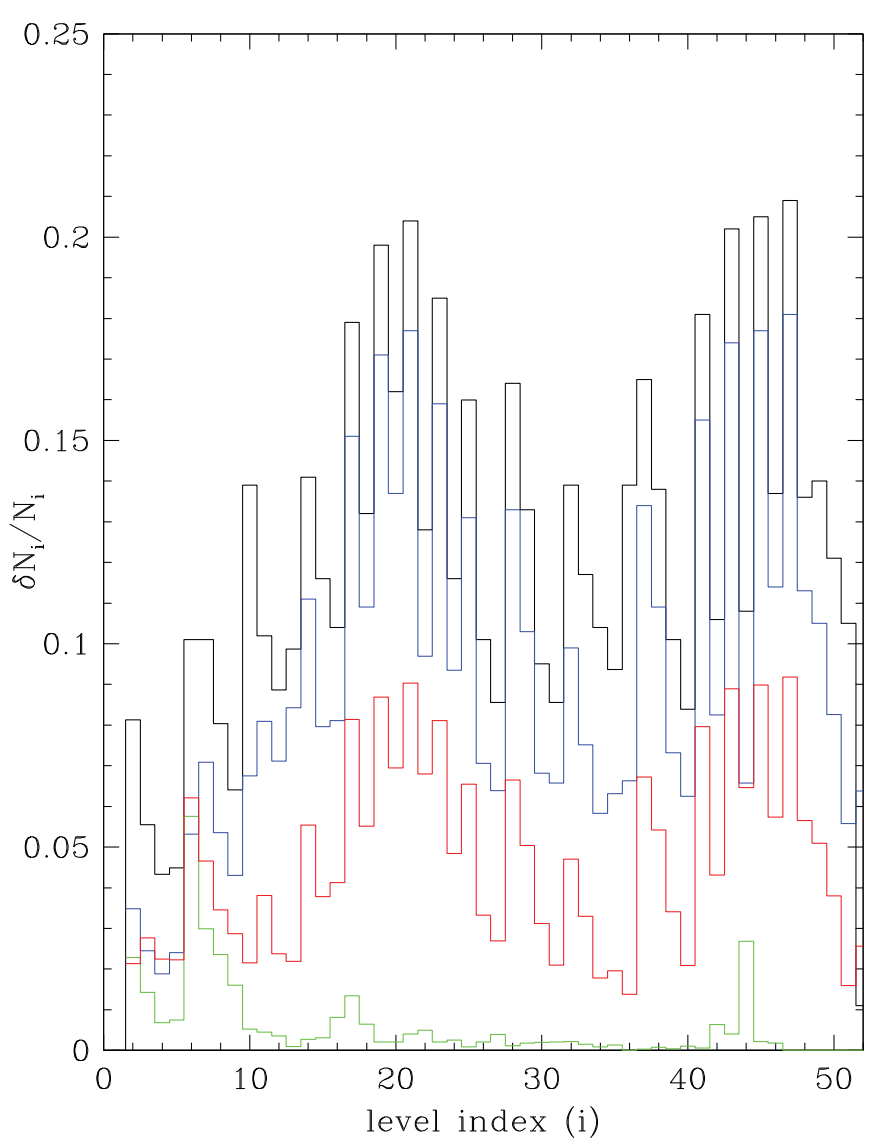

Figure 3. Estimated level population uncertainties for the lowest 52 levels of Fe II at $T_{e}=10^{4} \mathrm{~K}$ and $n_{e}=10^{4}$ (black line). Here we assume uncertainties for lifetimes, $A$-values, and collisional rates of $5 \%, 10 \%$, and $20 \%$, respectively. The figure also depicts the contributions from uncertainties in collisional rates (blue line), $A$-values (green line), and coupling of uncertainties among all levels (red line).

strongly coupled and uncertainties in atomic data are expected to propagate in a highly nonlinear fashion.

Figure 3 depicts the population errors for the lowest $52 \mathrm{lev}$ els of Fe II at $T_{e}=10^{4} \mathrm{~K}$ and $n_{e}=10^{4} \mathrm{~cm}^{-3}$. These are all even-parity metastable levels except for the ground level. The figure shows the total estimated uncertainties together with the direct contributions from uncertainties in the collision strengths and $A$-values (first and second terms on the right-hand side of Equation (2)) and the contribution from level uncertainty coupling. It is observed that the collision strengths are the dominant source of uncertainty for all levels except level $6\left(a^{4} F_{9 / 2}\right)$. For this level the uncertainty is dominated by the $A$-values and the uncertainty couplings with fine structure levels of the same multiplet and with levels of the ground multiplet. In turn, the $a^{4} F_{9 / 2}$ level makes the largest contribution to the uncertainties in 36 of the lowest 52 levels of Fe II. Unfortunately, the atomic data for the ${ }^{4} F_{9 / 2}$ level are among the most uncertain parameters of the whole Fe II system, as we discuss in the next section.

\section{ESTIMATING UNCERTAINTIES IN ATOMIC DATA}

In the previous section we adopted general uncertainties for lifetimes, $A$-values for forbidden transitions, and effective collision strengths of $5 \%, 10 \%$, and $20 \%$, respectively. In the absence of generally accepted procedures to estimate uncertainties in theoretical atomic data, these kinds of numbers are often cited in the literature as general guidelines; however, uncertainty estimates on specific rates are rarely provided. In Bautista et al. (2009) 

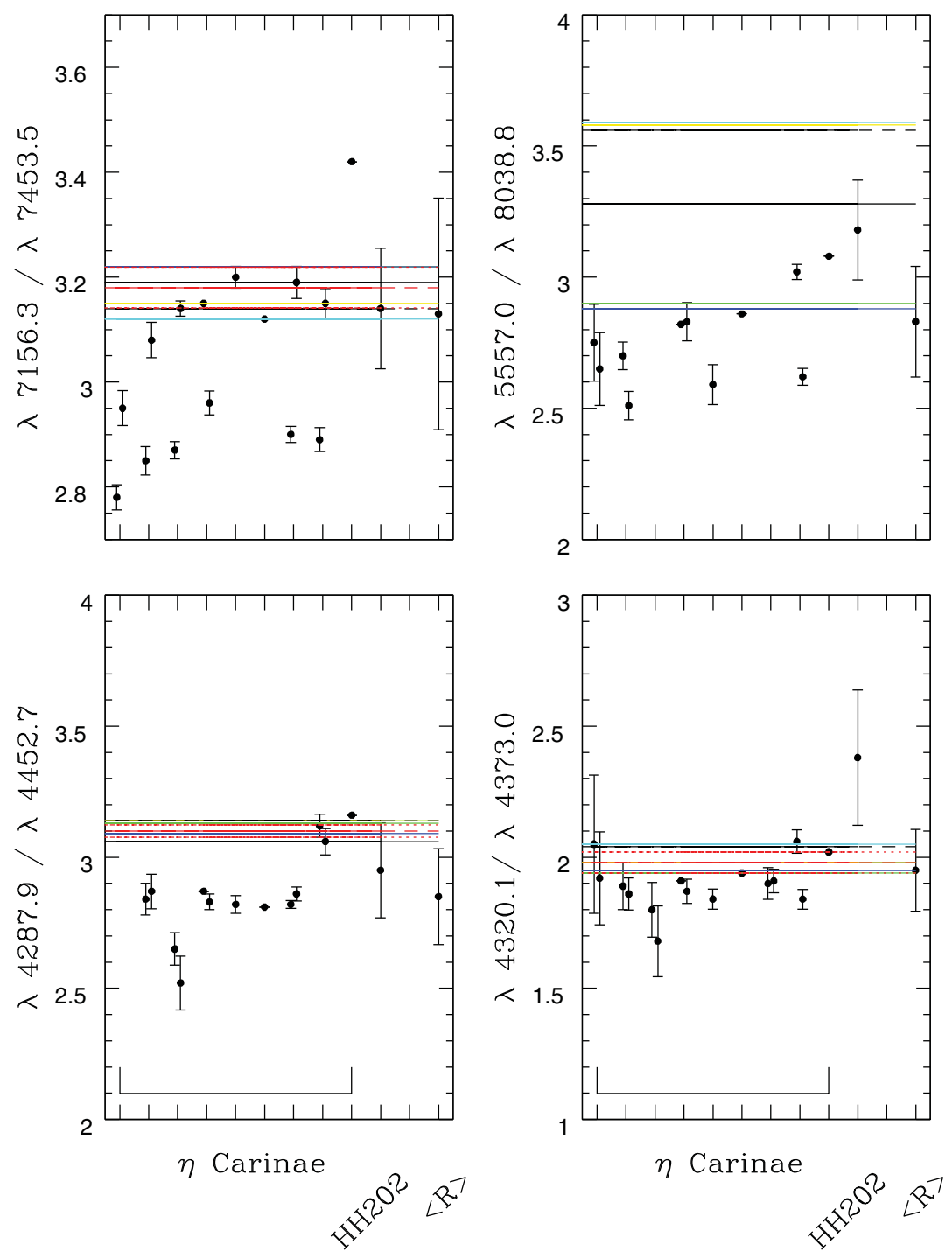

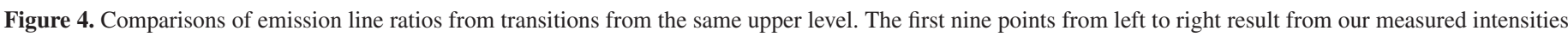

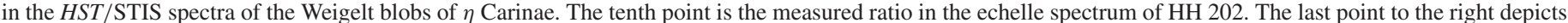

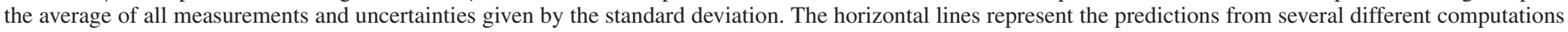
of $A$-values.

(A color version of this figure is available in the online journal.)

we proposed that uncertainties in $g f$-values could be estimated from the statistical dispersion among the results of multiple calculations with different methods and by different authors. The uncertainties can be constrained by comparing with experimental or spectroscopic data whenever available, although these also have significant associated uncertainties. This approach is similar to that carried out for many years by the Atomic Spectroscopy Data Center at the National Institute of Standards and Technology (NIST; http://www.nist.gov/pml/data/asd.cfm) in providing critical compilations of atomic data.

In estimating uncertainties from the dispersion of multiple results one must keep in mind some caveats. (1) Small scatter among rates is obtained when the computations converge to a certain value, although such a convergence is dependent on the maximum size of the quantum mechanical representation considered at the time of the computation. Thus, there is no guarantee that every seemingly converged result is indeed correct, as some values may result from local minima in the parameter space. (2) Large scatter among different calculations is expected in atomic rates where configuration interaction and level mixing lead to cancellation effects. The magnitude of these effects depends on the wave-function representation adopted. Therefore, some computations may be a lot more accurate than others for certain transitions and if we knew which computation is most accurate, then the scatter among all different results would overestimate the true uncertainty. However, detailed information about configuration and level mixing for every transition is rarely available in the literature. Thus, in the absence of complete information about every transition rate from every calculation, we propose that a practical and objective way to estimate uncertainties in atomic/molecular rates is by a critical comparison among the results from different calculations and experimental determinations, if available.

Does the statistical dispersion lead to realistic uncertainty estimates? To answer this question we look at the intensity ratios between emission lines from the same upper level as obtained from observed astronomical spectra and theoretical predictions. The advantage of looking at these ratios is that they depend only on the $A$-values, regardless of the physical conditions of the plasma. Thus, the ratios ought to be the same in the spectra 

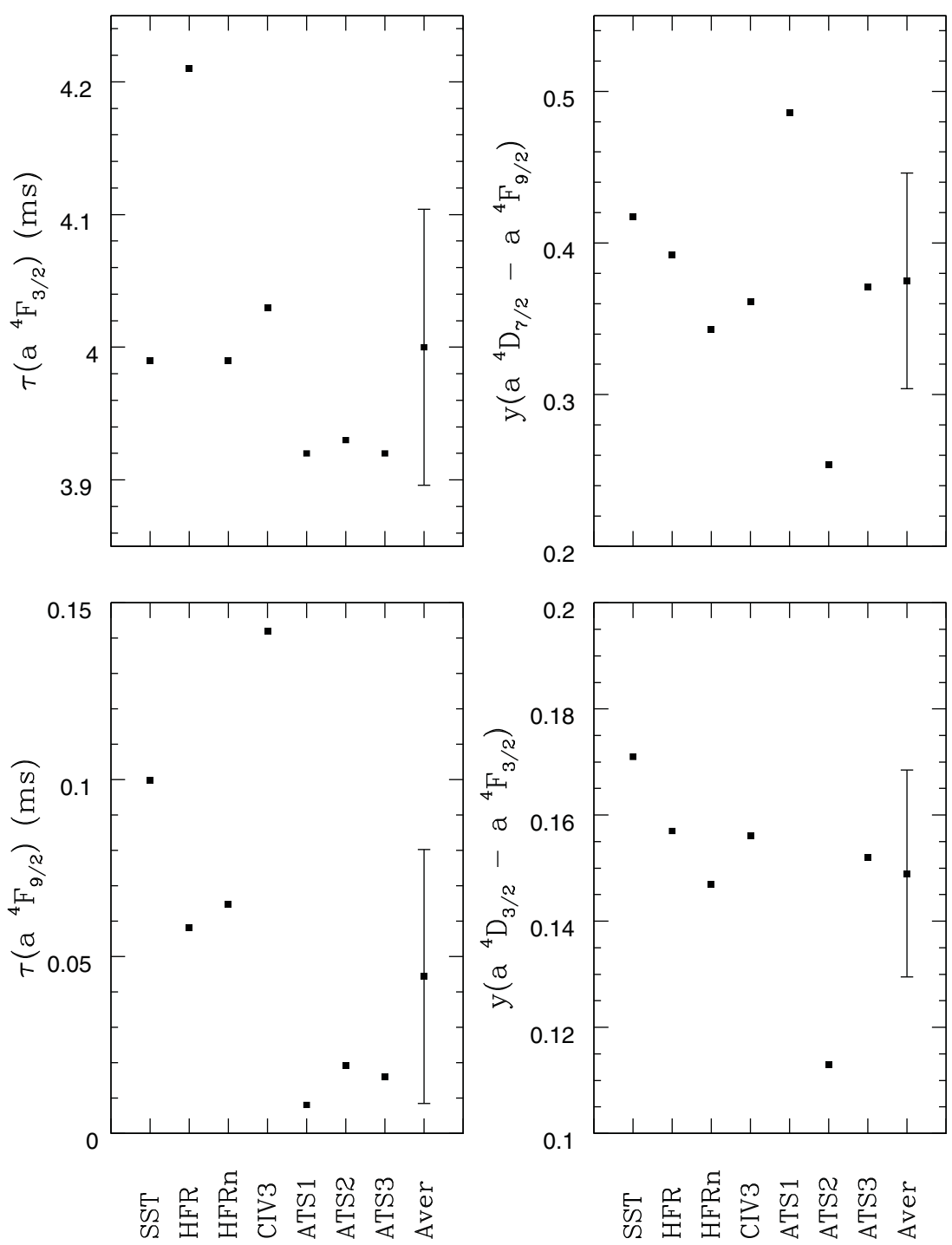

Figure 5. Theoretically calculated lifetimes and transition yields in Fe II. The calculations depicted are SST: SUPERSTRUCTURE computation by Quinet et al. (1996); HFR: HFR calculation also by Quinet et al.; HFRn: our new HFR calculation; CIV3: results by Deb \& Hibbert (2011); ATS21, ATS2, and ATS3: our new AUTOSTRUCTURE calculations that surpass Quinet et al. The last point to the right of each panel depicts the average value of the various determinations. The uncertainty bars for this point are set by the statistical dispersion between all values.

of any source, provided that the spectra have been corrected for extinction. Fe II yields the richest spectrum of all cosmic abundant chemical species. Thus, optical and near-IR [Fe II] lines are the best suited for the present experiment.

One hundred and thirty-seven [Fe II] lines are found in the $H S T /$ STIS archived spectra of the Weigelt blobs of $\eta$ Carinae. Six medium dispersion spectra $(R=6000$ to 10,000$)$ of the blobs were recorded between 1998 and 2004 at various orbital phases of the star's $5.5 \mathrm{yr}$ cycle. Seventy-eight [Fe II] lines are also present in the deep echelle spectrum $(R=30,000)$ of the Herbig-Haro object (HH 202) in the Orion nebula from Mesa-Delgado et al. (2009). The importance of having multiple spectra from different sources and different instruments must not be overlooked. Multiple measurements of the same line ratio minimize the likelihood of systematic errors due to unidentified blends, contamination from stellar emission, and instrumental effects.

From the observations, there are 107 line ratios well measured from the spectra. The ratios are defined as

$$
\text { ratio }=\max \left(F_{1}, F_{2}\right) / \min \left(F_{1}, F_{2}\right),
$$

where $F_{1}$ and $F_{2}$ are the measured fluxes of two lines from the same upper level. These ratios are defined to be always unconstrained above unity, so they are all equally weighted when comparing with theoretical expectations. Figure 4 illustrates a few line ratio determinations from several measurements from the spectra of $\eta$ Carinae and $\mathrm{HH} 202$, as well as from various theoretical determinations. Line flux measurements are done by fitting Gaussian profiles to each spectral line. The dispersion between the Gaussian profile and the observed feature determines the statistical uncertainty of each measurement. However, an additional, and often dominant, systematic uncertainty comes from the assumed continuum level within the spectral noise. Thus, we perform up to four measurements of every observation for different spectral extractions along the CCD and different assumptions about the continuum and the noise levels. We see that the scatter between multiple measurements of a given ratio greatly exceeds the statistical uncertainties. Moreover, the scatter between measured line ratios often exceeds the scatter between theoretical predictions. Full details about the Fe II spectra and measurement procedures will be presented in a forthcoming 


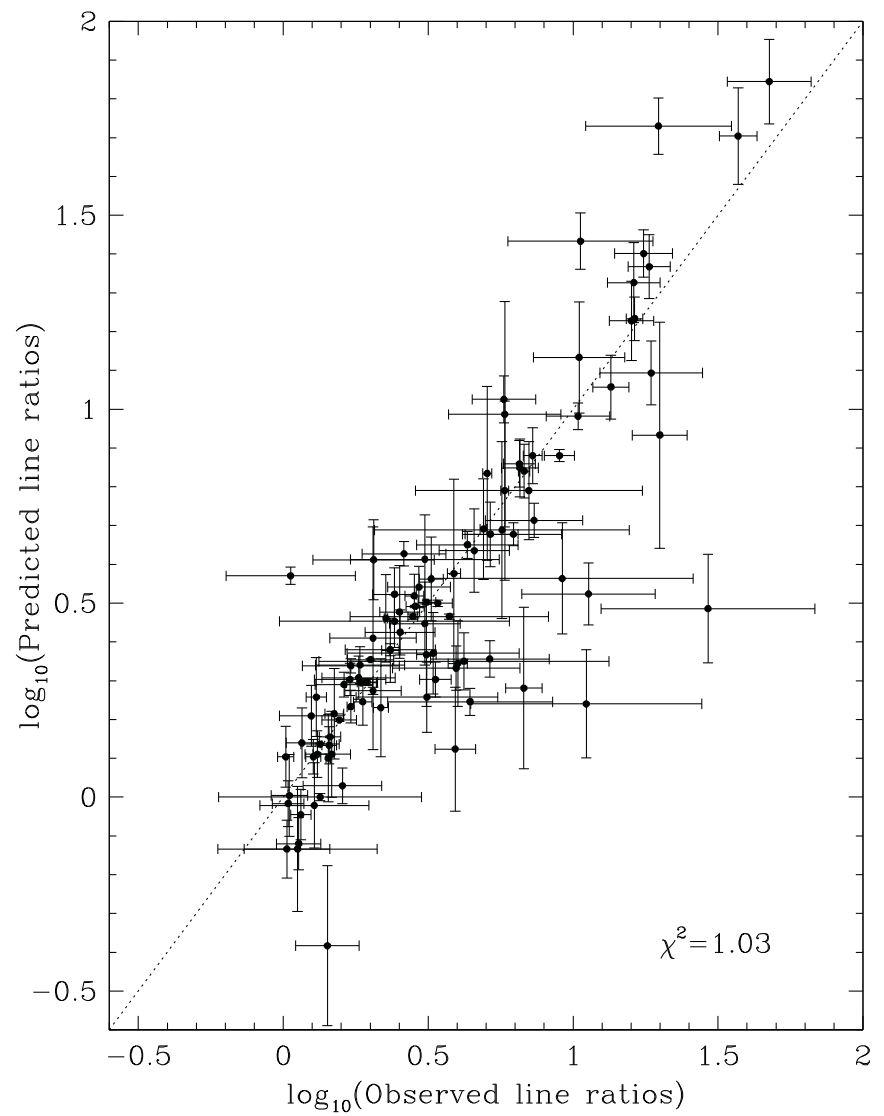

Figure 6. Line ratios between lines from the same upper level measured from optical nebular spectra vs. theoretical predictions.

paper, where we will also present our recommended atomic data for $\mathrm{Fe}$ II.

For the present work we consider seven different computations of $A$-values for Fe II. These are the SUPERSTRUCTURE and relativistic Hartree-Fock (HFR) calculations by Quinet et al. (1996), the recent CIV3 calculation of Deb \& Hibbert (2011), and various new HFR and AUTOSTRUCTURE calculations that extend previous works. Figure 5 presents a sample of theoretically calculated lifetimes and transition yields in Fe II. The yields are defined as $y_{i, j}=A_{i, j} \times \tau_{i}$. From the dispersion among various results, the average uncertainty in lifetimes for all levels of the $3 d^{7}$ and $3 d^{6} 4 s$ configuration is $13 \%$. More importantly, it is found that the uncertainty in the critically important $a^{4} F_{9 / 2}$ level is $\sim 80 \%$, due to cancellation effects in the configuration interaction representation of the $a^{4} F_{9 / 2}-a^{6} D_{9 / 2}$ transition.

We compared the observed line ratios described above with the predictions from different sets of theoretical $A$-values. Without uncertainty estimates for the theoretical values, the reduced$\chi^{2}$ values from these comparison range from 2.2 to 3100 for the different sets of $A$-values. On other hand, if one adopts average $A$-values from all calculations and uncertainties from the resultant standard deviations the reduced $-\chi^{2}$ is 1.03 . This is indicative of well estimated uncertainties, neither underestimated nor overestimated, and within these uncertainties there is good agreement between theoretical and experimental line ratios. The comparison between observed and theoretical line ratios, including uncertainties, is given in Figure 6.

Figure 7 shows the estimated lifetime uncertainties for the lowest 52 levels of Fe II. The figure also indicates the level population uncertainties that result from the present uncer-
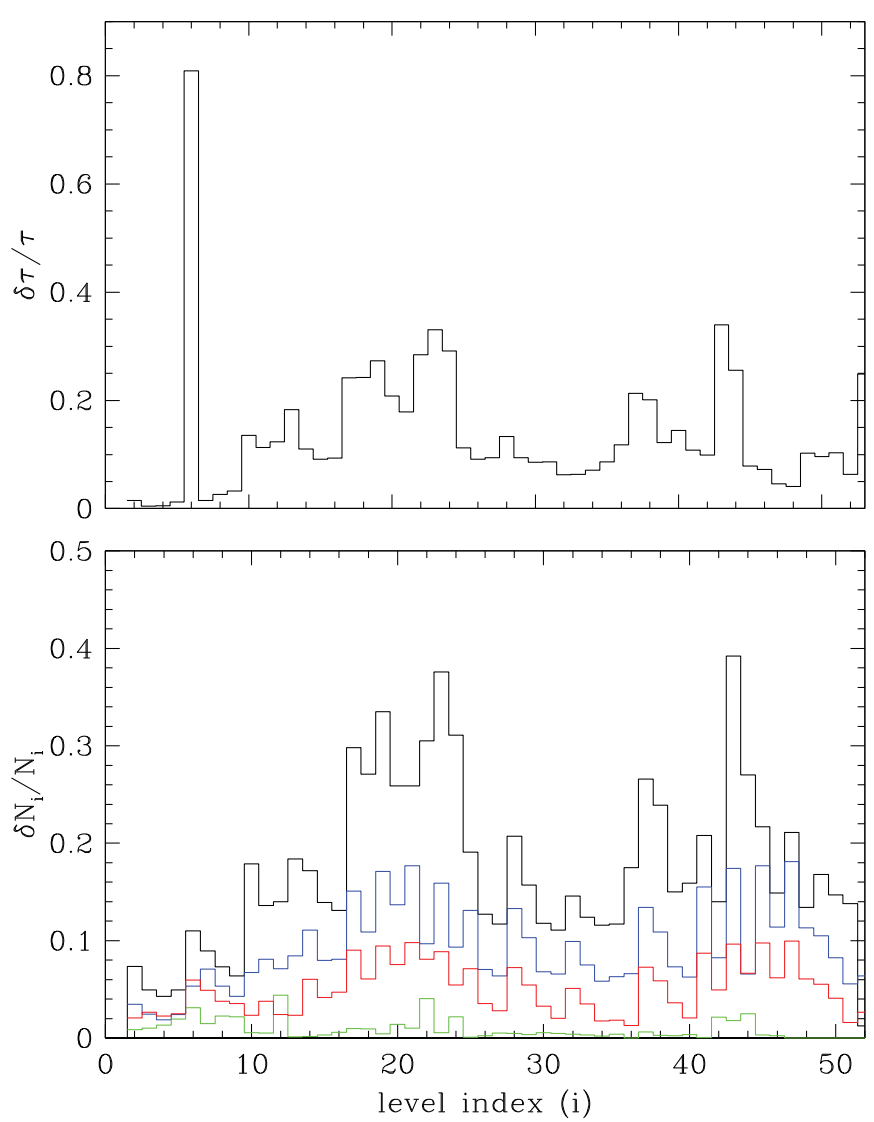

Figure 7. The upper panel presents the estimated uncertainties in lifetimes for the lowest 52 levels of Fe II. The lower panel is like Figure 3 but from uncertainties in lifetimes and radiative yields estimated from the dispersion among various calculations.

tainties in lifetimes and transition yields for a plasma with $T_{e}=10^{4} \mathrm{~K}$ and $n_{e}=10^{4} \mathrm{~cm}^{-3}$. Here, the adopted uncertainties in the collision strengths are kept at $20 \%$ for all transitions. The most uncertain lifetime is by far that of the important $a^{4} F_{9 / 2}$ level $(i=6)$, yet the way that this uncertainty propagates through level populations depends on the density of the plasma. For electron densities much lower than the critical density for the level the uncertainty in the lifetime correlated directly with the population for that level. This is seen at $n_{e}=10^{4} \mathrm{~cm}^{-3}$ for levels $\sim 18$ and higher. However, as the density increases, the uncertainties in the level populations become increasingly dominated by the collision strengths. This effect is clearly illustrated in Figure 8.

\section{UNCERTAINTIES IN EMISSION LINE EMISSIVITIES AND DIAGNOSTIC LINE RATIOS}

The line emissivity, in units of photons per second, of a transition $i \rightarrow f$, with $i>f$, is

$$
j_{i, f}=N_{i} \times A_{i, f}
$$

In computing the uncertainty in $j_{i, f}$ one must account for the fact that $N_{i}$ and $A_{i, f}$ are correlated, because the latter appears in the denominator term of Equation (1) that determines $N_{i}$. This is important because the most frequently observed lines from any upper level are usually those that dominate the total decay rate for the level, i.e., the inverse of the level's lifetime. It is 

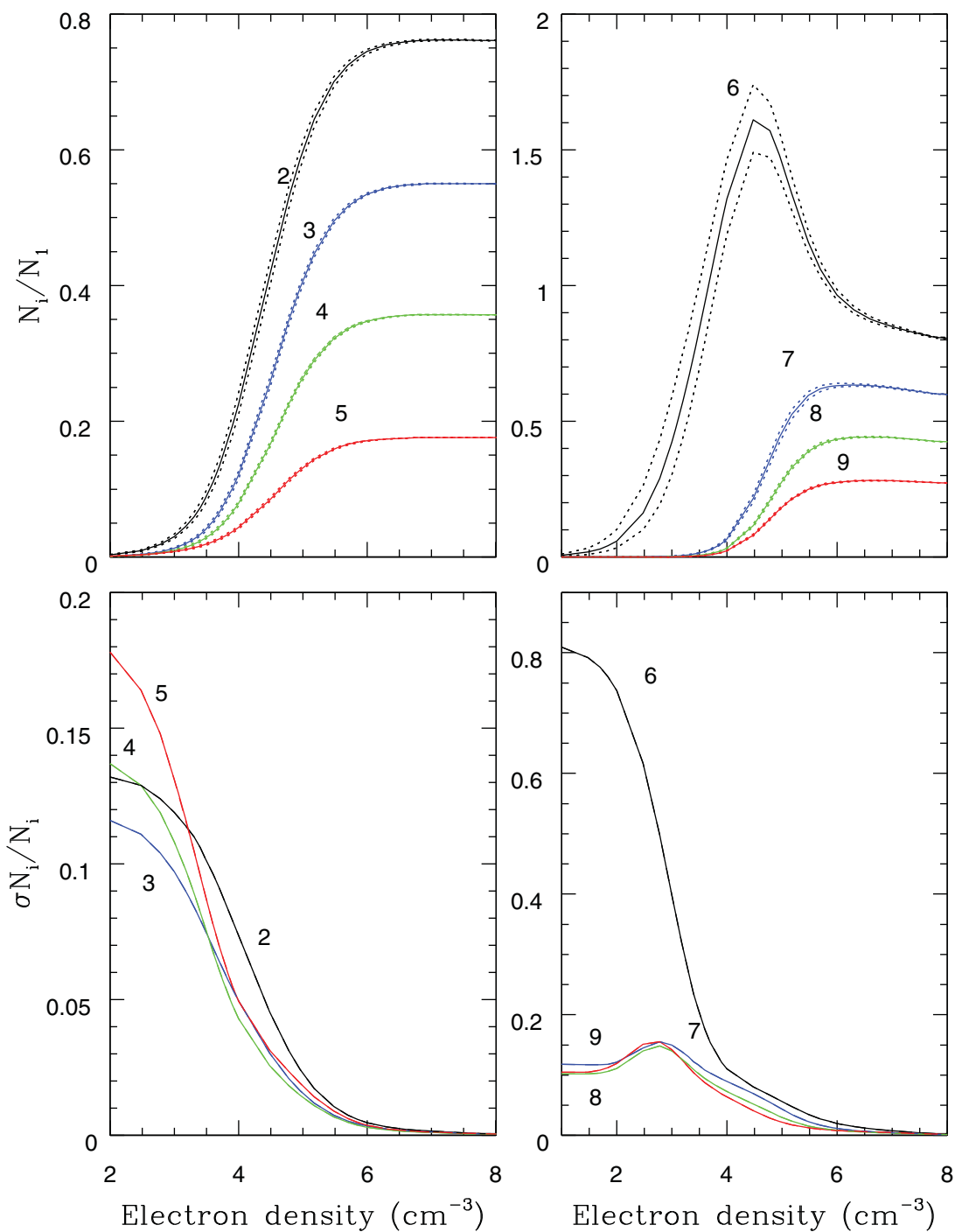

Figure 8. The same as Figure 2 but from uncertainties in lifetimes and radiative yields estimated from the dispersion among various calculations. (A color version of this figure is available in the online journal.)

convenient to rewrite the above equation as

$$
j_{i, f}=\kappa_{i} \frac{A_{i, f}}{n_{e} \sum_{j} q_{i, j}+\sum_{j} A_{i, j}} .
$$

Combining this equation with Equation (2) one finds

$$
\begin{aligned}
\left(\frac{\delta j_{i, f}}{j_{i, f}}\right)^{2}= & \left(\frac{\delta N_{i}}{N_{i}}\right)^{2}-\left(\frac{N_{i}}{\kappa_{i} \tau_{i}}\right)^{2}\left(\frac{\delta \tau_{i}}{\tau_{i}}\right)^{2} \\
& +\left(1-\frac{N_{i}}{\kappa_{i}} A_{i, f}\right)^{2}\left(\frac{\delta A_{i, f}}{A_{i, f}}\right)^{2} .
\end{aligned}
$$

This equation can be readily evaluated from the level populations and uncertainties already known. The equation has various interesting properties: (1) the equation is independent of the physical units used for the emissivities; (2) in the high-density limit, as the uncertainty in the level population goes to zero, the uncertainty in the emissivity is the same as that in $A$-value.

Figure 9 depicts uncertainties in emissivity for a sample of strong IR, near-IR, and optical [Fe II] lines. These are computed at $10^{4} \mathrm{~K}$. The uncertainties in the collision strengths are $20 \%$ and the uncertainties in the lifetimes and $A$-values are those estimated in the previous section. The behavior of these uncertainties for different physical conditions is complex. Let us consider, for instance, the uncertainty of emissivity of the $5.3 \mu \mathrm{m}$ line $\left(a^{4} F_{9 / 2}-a{ }^{6} D_{9 / 2} ; 6 \rightarrow 1\right)$ whose behavior is the inverse of the uncertainty in the population of the $a^{4} F_{9 / 2}$ level (see Figure 8). According to Equations (2) and (4), in the lowdensity limit

$$
j_{i, f} \rightarrow \sum_{k} N_{k} n_{e} q_{k, i}\left(\frac{A_{i, f}}{\sum_{j<i} A_{i, j}}\right) .
$$

In the case of the $a^{4} F_{9 / 2}$ level the $5.3 \mu \mathrm{m}$ transition dominates the total level decay rate and the ratio $A_{i, f} / \sum_{j<i} A_{i, j}$ is essentially 1 . Thus, the uncertainty in the $A_{6,1}$ rate cancels out at low electron densities and the uncertainty in the emissivity is small despite a large uncertainty in the level population. By contrast, at high densities the population of the level approaches the Boltzmann limit and the uncertainty in the emissivity is solely given by that in $A_{6,1}$, namely $\sim 80 \%$.

The line emissivity ratio between two lines is given by

$$
R=\frac{j_{i, f}}{j_{g, h}}=\left(\frac{N_{i}}{N_{g}}\right)\left(\frac{A_{i, f}}{A_{g, h}}\right)\left(\frac{\Delta E_{i, f}}{\Delta E_{g, h}}\right),
$$



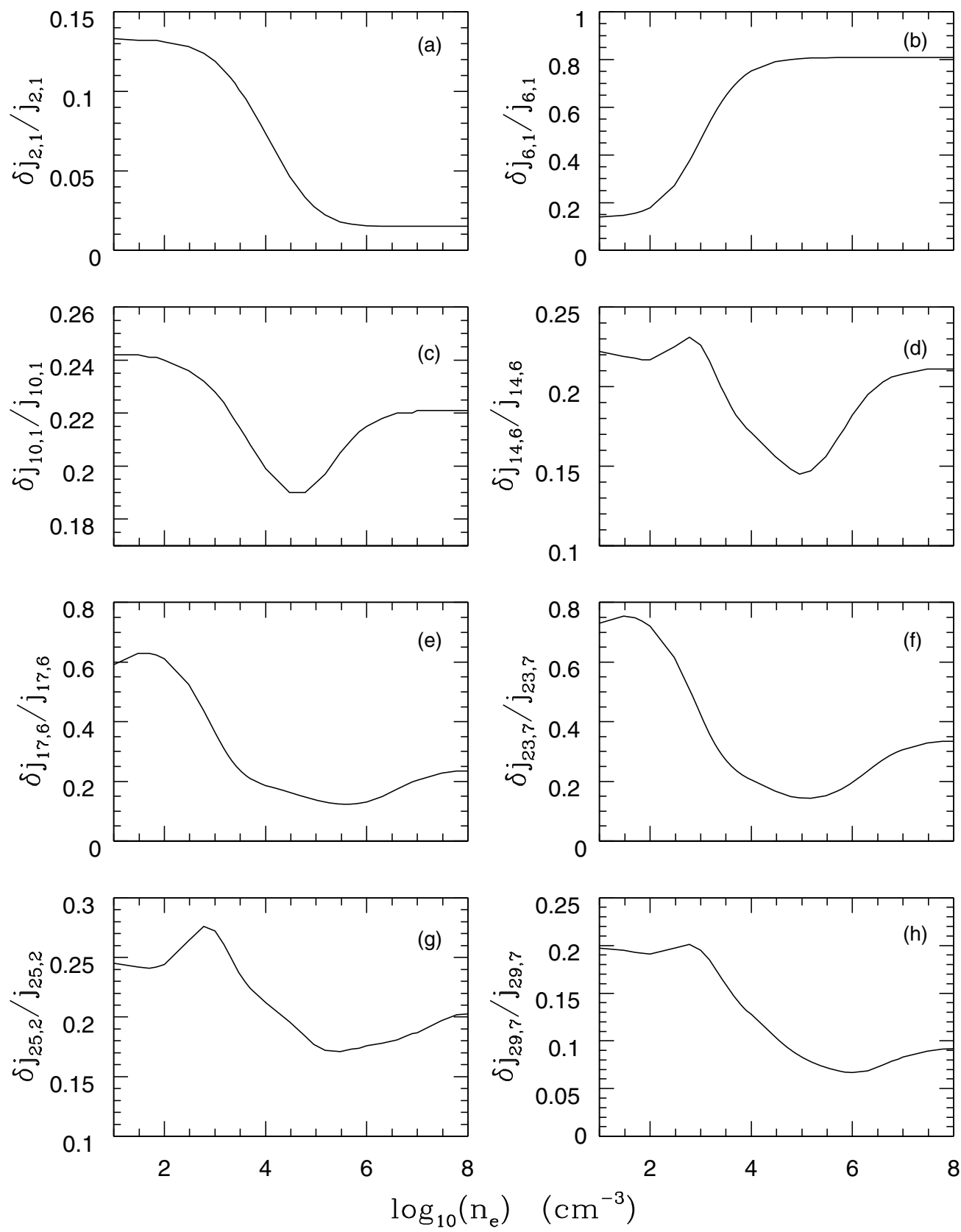

Figure 9. Uncertainties in [Fe II] line emissivities at $10^{4}$ vs. $n_{e}$. The transitions shown are (a) $25.9 \mu \mathrm{m}\left(a^{6} D_{7 / 2}-a{ }^{6} D_{9 / 2}\right)$; (b) $5.33 \mu \mathrm{m}\left(a^{4} F_{9 / 2}-a{ }^{6} D_{9 / 2}\right)$; (c) $1.256 \mu \mathrm{m}\left(a^{4} D_{7 / 2}-a{ }^{6} D_{9 / 2}\right)$; (d) $8616.8 \AA\left(a^{4} P_{5 / 2}-a^{4} F_{9 / 2}\right)$; (e) $7155.2 \AA\left(a^{2} G_{9 / 2}-a^{4} F_{9 / 2}\right)$; (f) $5527.4 \AA\left(a^{2} D_{5 / 2}-a^{4} F_{7 / 2}\right)$; (g) $4889.7 \AA\left(b^{4} P_{5 / 2}-a^{6} D_{7 / 2}\right)$; and (h) $5261.6 \AA\left(a^{4} H_{11 / 2}-a^{4} F_{7 / 2}\right)$.

where $\Delta E_{i, f}$ is the energy difference between levels $i$ and $f$ and we have used emissivities in units of energy per second. In computing the uncertainty in this line ratio one must account for the fact that the emissivities are correlated. Moreover, a general expression for the uncertainty must account for cases where $i=g$, in which case the uncertainty in the ratio would depend only on the $A$-values. The uncertainty is the ratio is given by

$$
\begin{aligned}
\left(\frac{\delta R}{R}\right)^{2}= & {\left[1-R\left(\frac{\partial j_{g, h}}{\partial j_{i, f}}\right)\right]^{2}\left(\frac{\delta j_{i, f}}{j_{i, f}}\right)^{2} } \\
& +\left[1-R\left(\frac{\partial j_{i, f}}{\partial j_{g, h}}\right)\right]^{2}\left(\frac{\delta j_{g, h}}{j_{g, h}}\right)^{2},
\end{aligned}
$$

where

$$
\frac{\partial}{\partial j_{i, f}}=\frac{1}{A_{i, f}} \frac{\partial}{\partial N_{i}}+\frac{1}{N_{i}} \frac{\partial}{\partial A_{i, f}} .
$$

Thus,

$$
\begin{aligned}
& \left(\frac{\delta R}{R}\right)^{2} \\
& =\left[1-R\left(\frac{A_{g, h} \Delta E_{g, h}}{A_{i, f} \Delta E_{i, f}} \frac{\partial N_{g}}{\partial N_{i}}+\frac{A_{g, h} \Delta_{g, h}}{\Delta E_{i, f} N_{i}} \frac{\partial N_{g}}{\partial A_{i, f}}\right)\right]^{2}\left(\frac{\delta j_{i, f}}{j_{i, f}}\right)^{2} \\
& +\left[1-R\left(\frac{A_{i, f} \Delta E_{i, f}}{A_{g, h} \Delta E_{g, h}} \frac{\partial N_{i}}{\partial N_{g}}+\frac{A_{i, f} \Delta_{i, f}}{\Delta E_{g, h} N_{g}} \frac{\partial N_{i}}{\partial A_{g, h}}\right)\right]^{2}\left(\frac{\delta j_{g, h}}{j_{g, h}}\right)^{2} .
\end{aligned}
$$



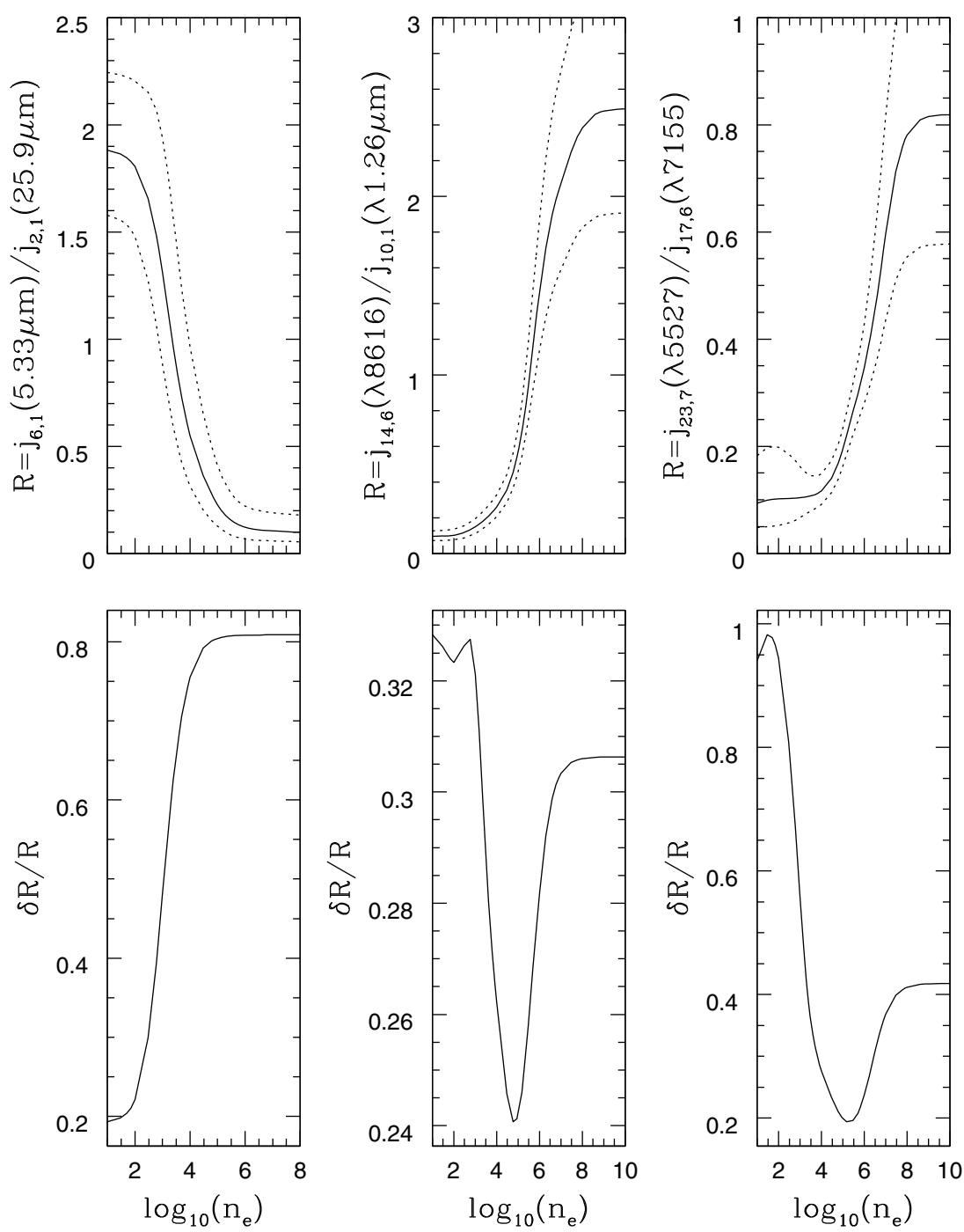

Figure 10. [Fe II] emissivity line ratios (upper panel) and uncertainties (lower panel) at $10^{4} \mathrm{~K}$ vs. electron density.

From Equation (2) we find $\left(\partial N_{i} / \partial A_{g, h}\right)=N_{i} N_{g} / \kappa_{i}$ for $h=i$, $\left(\partial N_{i} / \partial A_{g, h}\right)=-N_{i}^{2} / \kappa_{i}$ for $g=i$, and $\left(\partial N_{i} / \partial A_{g, h}\right)=0$ otherwise.

In the general case of a ratio involving several lines in the numerator and/or denominator, i.e.,

$$
R=\frac{\sum_{\{i, f\}} j_{i, f}}{\sum_{\{g, h\}} j_{g, h}}
$$

the uncertainty is

$$
\begin{aligned}
& \left(\frac{\delta R}{R}\right)^{2} \\
& =\sum_{\{i, f\}}\left(\frac{\sum_{\{i, f\}^{\prime}}\left(\partial j_{\{i, f\}^{\prime}} / \partial j_{\{i, f\}}\right)}{\sum_{\{i, f\}} j_{\{i, f\}}}-\frac{\sum_{\{g, h\}}\left(\partial j_{\{g, h\}} / \partial j_{\{i, f\}}\right)}{\sum_{\{g, h\}} j_{\{g, h\}}}\right)^{2}\left(\delta j_{\{i, f\}}\right)^{2} \\
& \times \sum_{\{g, h\}}\left(\frac{\sum_{\{g, h\}^{\prime}}\left(\partial j_{\{i, f\}^{\prime}} / \partial j_{\{g, h\}}\right)}{\sum_{\{g, h\}} j_{\{g, h\}}}-\frac{\sum_{\{g, h\}^{\prime}}\left(\partial j_{\{g, h\}^{\prime}} / \partial j_{\{g, h\}}\right)}{\sum_{\{g, h\}} j_{\{g, h\}}}\right)^{2}\left(\delta j_{\{g, h\}}\right)^{2} .
\end{aligned}
$$

Figure 10 shows a sample of line ratios between IR and optical lines and their uncertainties. The uncertainties exhibit complex behavior with variations in density and temperatures. In general, line ratios are only useful as diagnostics when the observed ratio lies around the mid-range of the theoretical ratio. Moreover, it is very important to know the uncertainties in the ratios when selecting appropriate diagnostics from a given spectrum.

\section{CONCLUSIONS}

We have presented a method to compute uncertainties in spectral models from uncertainties in atomic/molecular data. Our method is efficient and allows us to compute uncertainties in all level populations by solving a single algebraic equation. Specifically, we have treated the case of non-LTE models where electron impact excitation is balanced by spontaneous radiative decay. However, the method can be extended to ionization balance and additional excitation mechanisms.

Our method is tested in O III and Fe II models, first by assuming commonly assumed uncertainties and then by adopting uncertainties in lifetimes and $A$-values given by the dispersion between the results of multiple independent computations. Moreover, we show that uncertainties from the latter approach give in practice very good estimates.

Then we derive analytic expressions for the uncertainties in line emissivities and line ratios. These equations take into account the correlations between level populations and line emissivities. Interestingly, the behaviors of uncertainties in level 
populations and uncertainties in emissivities for transitions from the same upper levels are often different and even inverse. This is the case, in particular, for lines that result from transitions that dominate the total decay rate of the upper level. Then, the uncertainties in $A$-values for the transitions that yield the lines cancel out with the uncertainties in the lifetimes. In terms of emission line ratios, it is also found that knowledge of the uncertainties in the ratios is essential in selecting appropriate ratios for density and temperature diagnostics.

At present, we are in the process of estimating uncertainties in atomic data for species of astronomical interest. Our uncertainty estimates and analysis in various spectral models, ionic abundance determinations, and diagnostic line ratios will be presented elsewhere.

We acknowledge financial support from grants from the NASA Astronomy and Physics Research and Analysis Program (award NNX09AB99G). The Belgian FRS-FNRS is also acknowledged.

\section{REFERENCES}

Ballance, C. P., Loch, S. D., Foster, A. R., et al. 2013, Fusion Sci. Technol., submitted

Bautista, M. A., \& Pradhan, A. K. 1998, ApJ, 492, 650

Bautista, M. A., Quinet, P., Palmeri, P., et al. 2009, A\&A, 508, 1527

Deb, N. C., \& Hibbert, A. 2011, A\&A, 536, A74

Dunn, J. P., Bautista, M., Arav, N., Moe, M., \& Korista, K. 2010, ApJ, 709,611

Luridiana, V., \& García-Rojas, J. 2012, in IAU Symp. 283, Planetary Nebulae: An Eye to the Future (Cambridge: Cambridge Univ. Press), 139

Luridiana, V., García-Rojas, J., Aggarwal, K., et al. 2011, arXiv:1110.1873

Mesa-Delgado, A., Esteban, C., García-Rojas, J., et al. 2009, MNRAS, 395,855

Quinet, P., Le Dourneuf, M., \& Zeippen, C. J. 1996, A\&AS, 120, 361

Wesson, R., Stock, D. J., \& Scicluna, P. 2012, MNRAS, 422, 3516 